the Johns-Manville Corporation assigned to the new Manville Corporation only those assets associated with asbestos, thus shielding $\mathbf{7 4}$ per cent of its total assets from future litigation. It has even argued that claims against the Johns-Manville Corporation are not valid because that corporation no longer exists.

Other companies have been doing much the same. The Union Asbestos and Rubber Company became Unarco Industries Inc. and then UNR Industries. It has not made asbestos products since 1962 , but claims are still pouring in. When it created UNR Industries Inc., it assigned to that company only its pre-1970 assets, which amounted to $\$ 24$ million. (Its assets today are $\$ 200$ million.) Then UNR Industries Inc. filed for bankruptcy in Chicago on the basis of the intolerable outstanding asbestos claim, so perhaps inspiring the Manville Corporation to do the same. Deborah Shapley

\section{NRDC and NEB}

\section{Union delayed}

The National Research Development Corporation is still coining money from the cephalosporin antibiotics, synthetic pyrethroid insecticides and half a dozen other inventions, but its intended marriage with the National Enterprise Board, announced in July 1981, has been delayed. Sir Freddie Wood, chairman of both organizations (which also now have identical boards of directors), said last week that he hopes to know more about the British government's intentions in a few months, but that legislation is unlikely to see the light of day before the end of the next year-long session of the British Parliament, beginning next week.
Meanwhile, the common opinion is that a formal merger of the two organizations would indeed bring the benefits advertised for it. The corporation, set up in 1949 to exploit inventions arising in the public sector (including universities) has been run principally by technical people, and during its chequered history has been accused of neglecting commercial opportunities. The board, by contrast, is stronger on accountancy and financial management.

The corporation as a single entity seems in good financial shape, to judge from the annual report published a week ago. It earned $£ 26.2$ million in the year to 31 March, chiefly from royalties on licensed inventions. Payments to inventors (and their institutions) amounted to $£ 3.53$ million, rather less than the sum set aside by the corporation for taxation before reaching its net profit of $£ 5.02$ million.

In the years immediately ahead, however, the corporation's income is likely to decline as patents on the cephalosporins run out. The annual report says that synthetic pyrethroids should help bridge the gap, but that these insecticides are unlikely to be as profitable. The cephalosporins stem from research at the University of Oxford, the pyrethroids from the Rothamsted Experimental Station.

During the year, the corporation says that 229 patents were assigned to it, a small increase on the previous year - and a surprisingly small number absolutely. The total of inventions about which the corporation was told amounted to 1,935 , well over half from the public sector. Something like 350 inventions were earning money at the end of the year, while $£ 23$ million had been committed (or spent) on the development of patented inventions and $£ 48$ million to joint development ventures with industrial companies.

\title{
Celltech eyes Japanese market
}

Celltech, the British biotechnology company, has concluded a major fiveyear agreement for exclusive distribution of its alpha-interferon purification and assay products with the Sumitomo Corporation of Japan. The deal also covers Celltech's monoclonal antibody ABO blood grouping reagents, as well as anti-beta and gamma interferons.

Sumitomo, one of Japan's largest trading companies, has been selling Celltech products on an informal basis for several months; now Celltech hopes to reach a dominant position in the Japanese pharmaceutical and diagnostics market, valued at $£ 8,500$ million per year. Celltech is planning to develop monoclonal antibodies that can be incorporated into diagnostic kits and also hopes to gain some contract research from the present agreement; other cooperative ventures are not excluded.

The agreement follows closely that between Biogen of Switzerland and Fujisawa for joint development of
Biogen's human tissue plasminogen activator, which has yet to reach the stage of clinical trials. Hopes are high for this anticoagulant protein, which is more specific in its effects than other thrombolytic agents now in use, urokinase for example. It may come to represent a significant fraction of the large Japanese market for such agents. Fujisawa already has agreements with overseas companies for the production of human interferons.

These agreements follow a trend which has become apparent over the past few years in which Japanese firms arrange joint development and distribution deals with European and US companies; such arrangements have been reached between Hoffmann-La Roche, Genentech and Takeda, for example. While the Japanese appear actively to be developing their own expertise in techniques such as the production of monoclonal antibodies and recombinant DNA, they seem also to be encouraging the import of foreign products.
Astrophysics laboratory Theoretical gain Chicago

The Fermi National Accelerator Laboratory (Fermilab) has set up the first theoretical astrophysics group to reside at any of the world's accelerator laboratories. The group will start work in January, and is being supported by the US National Aeronautics and Space Administration to the tune of $\$ 500,000$ over three years. By January, Dr Leon M. Lederman, director of Fermilab, expects to have taken on one or two senior researchers and two or three junior workers.

Lederman says: "One of the most exciting parts of particle physics research now is the connection between inner and outer space. Particle physicists look down with their microscopes and astrophysicists look up with their telescopes and find they are looking at some of the same things'".

Fermilab's studies are essential to model the evolution of the Universe immediately after the Big Bang, but Lederman says that the behaviour of the early Universe in turn constrains what the particle physicists can expect to find as accelerator energies increase.

The new group, with a huge accelerator readily available, will deal primarily with the connection between particle physics and the early Universe, in which high temperatures and energies were generated by the Big Bang. But current interests in particle physics research, the grand unified theories for example, deal with particle energies around $10^{14} \mathrm{GeV}$, ten orders of magnitude greater than the energies being dreamed of by today's accelerator builders.

Yet astrophysical theory can extrapolate as far back in time as $10^{-43}$ seconds after the Big Bang - when the thermal energy would have been $10^{19} \mathrm{GeV}$. The early Universe is thus an ersatz particle accelerator for extreme energies. The group will also investigate the implications of particle physics theory for astrophysics. Some grand unified theories suggest that the Universe passed through an "inflationary" phase of violent expansions that may explain why the Universe today is isotropic, homogeneous and dynamically "flat".

Lederman says that Fermilab has hitherto only "dabbled" in astrophysics, and he hopes that the new formal collaboration will enable more systematic and fruitful studies to be made.

Larry Arbeiter

\section{Ciba Foundation}

In Nature for 30 September, p. 383 , there was a report of what Dr R.G. Edwards had said at a Galton Lecture and at a meeting organized by the British Medical Journalists' Association; this meeting was sponsored by Ciba-Geigy Pharmaceuticals and not, as reported, by the Ciba Foundation. 